\title{
Impacto de la violencia homicida en la esperanza de vida masculina de México
}

\author{
Guillermo Julián González-Pérez, ${ }^{1}$ María Guadalupe Vega-López ${ }^{1}$ \\ y Carlos Enrique Cabrera-Pivaral ${ }^{1}$
}

Forma de citar

González-Pérez GJ, Vega-López MG, Cabrera-Pivaral CE. Impacto de la violencia homicida en la esperanza de vida masculina de México. Rev Panam Salud Publica. 2012;32(5):335-42.

RESUMEN Objetivo. Determinar el impacto de la violencia homicida en la esperanza de vida masculina (EVM) de México y sus 32 estados durante los trienios 1998-2000 y 2008-2010, así como el grado de participación de los diferentes grupos etarios en la pérdida de años de EVM por esta causa.

Métodos. A partir de datos oficiales sobre defunciones y población, se construyeron tablas abreviadas de mortalidad masculina para México y sus estados en los trienios estudiados. Se calcularon la esperanza de vida temporaria masculina y los años de esperanza de vida perdidos (AEVP) en el grupo etario de 15 a 75 años, por causas seleccionadas (homicidios, diabetes mellitus y accidentes de transporte) y por grupos de edad en cada trienio.

Resultados. Entre 1998-2000 y 2008-2010 se incrementaron los AEVP por homicidio a nivel nacional y en 19 estados; en cuatro de ellos, la cifra de AEVP en 2008-2010 superó los dos años, destacándose el caso de Chihuahua, con 5,2 años. De 18 estados donde la esperanza de vida temporaria masculina disminuyó entre los dos trienios, en 14 aumentaron los AEVP por homicidio. En 2008-2010 los homicidios fueron responsables del mayor porcentaje de AEVP masculinos en el grupo etario de 20 a 44 años. Los AEVP por homicidio en el grupo de 15 a 44 años de edad se incrementaron entre ambos trienios.

Conclusiones. El aumento de la violencia homicida, especialmente entre los jóvenes, es un freno para el incremento de la esperanza de vida masculina en México. En varios estados, como Chihuahua y Durango, esta violencia parece ser la principal responsable del descenso de la esperanza de vida masculina entre los 15 y 75 años de edad.

Palabras clave Violencia; homicidio; mortalidad; esperanza de vida; México.

El aumento de la esperanza de vida al nacer (EVN) en toda América Latina ha sido considerable en los últimos 60 años (1). En el caso particular de México, pasó de 49,6 años para ambos sexos en 1950 a 75,3 años en 2010, un incremento de más de 25 años en seis décadas. En los hombres, la EVN subió de 48,1 años a 73,1 en el mismo lapso (2). Sin embargo, entre 1995 y 2010 el incremento de la EVN ha

\footnotetext{
1 Universidad de Guadalajara, Ciencias Sociales, Guadalajara, México. La correspondencia se debe dirigir a Guillermo González-Pérez. Correo electrónico: ggonzal@cencar.udg.mx
}

sido pequeño - de 73,6 a 75 años la EVN general y de 71,3 a 73,1 la masculina-, lo cual refleja una ralentización en la tendencia al descenso de la mortalidad observada en décadas pasadas a nivel nacional.

Es bien conocida la mayor probabilidad de morir por causas violentas que tienen los hombres con respecto a las mujeres (3). En el contexto mexicano, donde se ha observado recientemente un notable crecimiento de la violencia interpersonal, sobre todo de homicidios (4), la tasa de homicidios masculina ha ascendido de forma notoria (de 15,4 por 100000 habitantes en 2007 a 33,4 en 2010), decuplicando la correspondiente tasa femenina en este último año (5): una sobremortalidad masculina por este tipo de violencia comparable a la de Colombia (6), pero mucho mayor a la que registran Estados Unidos y Canadá $(7,8)$

Los niveles de violencia, y sobre todo las tasas de homicidio, son en buena parte manifestaciones de graves problemas sociales que incluyen, entre otros, el crimen organizado, la corrupción, la impunidad y las desigualdades sociales (9-11). En 
este sentido, el comportamiento de las tasas de homicidio masculinas -y su impacto en la esperanza de vida- variarán de acuerdo con las disparidades y las condiciones socioeconómicas imperantes en una región o país determinados (12).

A nivel internacional, al igual que en México, son más bien escasos los trabajos que han estimado el efecto de los homicidios en la esperanza de vida. Lo destacable es que todos ellos coinciden en afirmar que los homicidios desempeñan un importante papel en la pérdida de años de esperanza de vida, en especial entre los hombres (12-15). El presente estudio tiene como propósito determinar el impacto de la violencia homicida en la esperanza de vida masculina (EVM) de México y sus 32 estados durante los trienios 1998-2000 y 2008-2010, así como el grado de participación de los diferentes grupos etarios en la pérdida de años de EVM por esta causa.

\section{MATERIALES Y MÉTODOS}

Se realizó un estudio observacional, de carácter transversal y descriptivo, basado en fuentes secundarias de información. Los datos sobre mortalidad para cada trienio estudiado fueron obtenidos de las bases de datos oficiales del Sistema Nacional de Información en Salud (SINAIS) de la Secretaría de Salud (16), mientras que los datos sobre población se obtuvieron de la proyección de población elaborada por el Colegio de México (17) para el país y cada estado entre 1990 y 2012.

La elección de los trienios 1998-2000 y 2008-2010 responde tanto al hecho de poder trabajar con datos referidos a una sola revisión (la décima) de la Clasificación Internacional de Enfermedades (CIE-10) (18), como a la posibilidad de comparar la situación más reciente del país con lo sucedido 10 años atrás. En cada trienio se analizaron todas las defunciones masculinas registradas: 741165 entre 1998 y 2000 y 948922 entre 2008 y 2010, así como aquellas debidas a homicidios (32 385 y 53698 en 1998-2000 y 2008-2010, respectivamente), diabetes mellitus (59 742 y 112 385) y accidentes de transporte (34 990 y 41 845).

De las defunciones masculinas analizadas, hubo 4656 en 1998-2000 (0,6\% del total) y 5572 en 2008-2010 (0,6\% del total) sin edad registrada. En el caso de los homicidios, las defunciones de edad no especificada fueron $522(1,6 \%$ del total) en 1998-2000 y 1297 (2,4\% del total) en 2008-2010 (16). Para corregir esta situación, se procedió a redistribuir proporcionalmente las defunciones de edad ignorada - en general y de manera específica por cada causa examinadasegún el peso relativo de cada grupo etario, a nivel nacional y en cada estado. Este tipo de redistribución se emplea con frecuencia en estudios de mortalidad, no altera el sentido de los hallazgos y se basa en los principios de expansión del muestreo aleatorio simple (19-22).

Todas las causas investigadas se clasificaron en base a la CIE-10: homicidios (X85-Y09, Y87.1), diabetes mellitus (E10-E14) - primera causa de defunción en México- y accidentes de transporte (V01-V99x), la causa de muerte violenta que en años recientes ha provocado más fallecimientos entre los hombres mexicanos (16).

A partir de la información existente sobre defunciones y población, se construyeron tablas de mortalidad masculinas abreviadas para México, para el Distrito Federal y para los 31 estados que conforman el país en cada trienio; para la construcción de las tablas de mortalidad se utilizó el modelo oeste de las tablas desarrolladas por Coale y Demeny (23), recomendado para países con niveles de mortalidad semejantes al de México $(24,25)$. La construcción de tablas de mortalidad trienales tuvo el objeto de reducir posibles variaciones aleatorias de la mortalidad que pudieran distorsionar la tendencia de su comportamiento (24).

El método utilizado permitió calcular los años de esperanza de vida perdidos (AEVP) en general, por causa - homicidios, diabetes mellitus y accidentes de transporte- $-y$ por grupos de edad en cada trienio, según el método propuesto por Arriaga $(14,26,27)$. En términos prácticos, los AEVP representan la diferencia entre el máximo posible de años que se pueden vivir entre dos edades y la esperanza de vida temporaria, que es el promedio de años vividos por la población entre esas edades (26); el número de AEVP se calcula multiplicando la proporción de personas que fallecen entre las edades $x$ y $x+n$ en la población estacionaria de la tabla de mortalidad, por la diferencia entre el número medio de años que podrían haber vivido desde $x$ en caso de no fallecer y el promedio de años realmente vividos entre $x$ y $x+n$ por la población que muere en ese grupo de edad (24).
De este modo, se calcularon AEVP para la población entre 15 y 75 años, suponiendo la existencia de una mortalidad nula entre ambas edades. Este supuesto facilita la interpretación del indicador: el total de AEVP entre las edades "15" y "75" es igual a la diferencia entre " 75 " y " 15 " (o sea, el número máximo de años que se pueden vivir entre ambas edades si se elimina la mortalidad [en este caso, 60 años] y la esperanza de vida temporaria entre "15" y "75" [es decir, el promedio de años reales vividos]). La elección de este rango de edades se debe a que comprende el principal período de actividad económica de la población, no toma en cuenta la mortalidad en los dos extremos de la estructura de edades, cuya dinámica presenta características propias, y es un período de la vida cuando las personas tienen suficiente independencia como para que su comportamiento pueda tener alguna repercusión, como es el caso de las muertes violentas. Además, permite la comparación con datos previos de México y otros países latinoamericanos (14).

Para la construcción de tablas de mortalidad, así como para el cálculo de AEVP, se utilizó el programa EPIDAT v3.1 (28).

\section{RESULTADOS}

A nivel nacional, en 2008-2010 la diabetes fue responsable de $11,8 \%$ de los AEVP por hombres de entre 15 y 75 años de edad, y los homicidios de $11,7 \%$, en ambos casos un porcentaje mayor al que representaron los accidentes de transporte $(7,9 \%)$ (cuadro 1 ).

En la mayoría de los estados del país, la diabetes tuvo más peso en cuanto a AEVP masculinos que los homicidios y los accidentes de transporte. Sin embargo, hay nueve estados (Baja California, Chihuahua, Durango, Guerrero, Michoacán, Nayarit, Oaxaca, Sinaloa y Sonora) donde los homicidios fueron la causa principal de pérdida de años de EVM, destacando casos como Chihuahua, donde representó más del $40 \%$ de todos los AEVP masculinos en 2008-2010, Sinaloa, que aportó un tercio, y Guerrero y Durango, donde representó algo más de la cuarta parte. Entre 19982000 y 2008-2010, los AEVP por homicidio en el sexo masculino a nivel nacional pasaron de 0,58 a 0,84 , un incremento relativo anual de $3,7 \%$; a su vez, la EVM temporaria creció 0,05 años entre ambos 
CUADRO 1. Años de esperanza de vida perdidos (AEVP), por causas seleccionadas y por estado, en personas de entre 15 y 75 años, México, 2008-2010

\begin{tabular}{|c|c|c|c|c|c|c|}
\hline Estado & Homicidios & $\begin{array}{c}\text { Diabetes } \\
\text { mellitus }\end{array}$ & $\begin{array}{c}\text { Accidentes } \\
\text { de } \\
\text { transporte }\end{array}$ & $\begin{array}{c}\text { Otras } \\
\text { causas }\end{array}$ & $\begin{array}{l}\text { Total } \\
\text { AEVP }\end{array}$ & $\begin{array}{c}\text { AEVP } \\
\text { Homicidios } \\
(\%)\end{array}$ \\
\hline México (todos los estados) & 0,84 & 0,85 & 0,57 & 4,92 & 7,18 & 11,69 \\
\hline Aguascalientes & 0,29 & 0,82 & 0,77 & 4,38 & 6,26 & 4,69 \\
\hline Baja California & 1,68 & 0,70 & 0,25 & 5,68 & 8,30 & 20,23 \\
\hline Baja California Sur & 0,27 & 0,72 & 0,87 & 4,89 & 6,75 & 3,98 \\
\hline Campeche & 0,26 & 0,72 & 0,55 & 4,92 & 6,45 & 4,08 \\
\hline Coahuila & 0,55 & 1,02 & 0,49 & 4,90 & 6,96 & 7,96 \\
\hline Colima & 0,58 & 0,75 & 0,76 & 4,62 & 6,71 & 8,61 \\
\hline Chiapas & 0,32 & 0,65 & 0,25 & 6,03 & 7,24 & 4,36 \\
\hline Chihuahua & 5,23 & 0,68 & 0,66 & 5,44 & 12,01 & 43,52 \\
\hline Distrito Federal & 0,44 & 1,03 & 0,35 & 4,62 & 6,45 & 6,86 \\
\hline Durango & 2,60 & 0,70 & 0,86 & 4,94 & 9,11 & 28,50 \\
\hline Estado de México & 0,55 & 1,02 & 0,48 & 4,32 & 6,37 & 8,65 \\
\hline Guanajuato & 0,38 & 1,05 & 0,81 & 4,56 & 6,80 & 5,61 \\
\hline Guerrero & 2,29 & 0,73 & 0,50 & 4,73 & 8,25 & 27,77 \\
\hline Hidalgo & 0,23 & 0,80 & 0,56 & 5,35 & 6,94 & 3,37 \\
\hline Jalisco & 0,48 & 0,80 & 0,75 & 4,79 & 6,81 & 7,08 \\
\hline Michoacán & 0,97 & 0,84 & 0,89 & 5,07 & 7,76 & 12,45 \\
\hline Morelos & 0,87 & 0,89 & 0,44 & 4,48 & 6,67 & 12,97 \\
\hline Nayarit & 1,38 & 0,56 & 1,00 & 4,49 & 7,43 & 18,54 \\
\hline Nuevo León & 0,47 & 0,75 & 0,31 & 4,95 & 6,48 & 7,24 \\
\hline Oaxaca & 0,87 & 0,73 & 0,69 & 5,34 & 7,63 & 11,37 \\
\hline Puebla & 0,29 & 1,02 & 0,52 & 5,08 & 6,92 & 4,22 \\
\hline Querétaro & 0,20 & 0,82 & 0,79 & 4,71 & 6,51 & 3,00 \\
\hline Quintana Roo & 0,43 & 0,72 & 0,46 & 4,90 & 6,51 & 6,57 \\
\hline San Luis Potosí & 0,48 & 0,74 & 0,67 & 4,39 & 6,28 & 7,67 \\
\hline Sinaloa & 2,80 & 0,49 & 0,84 & 4,18 & 8,30 & 33,67 \\
\hline Sonora & 0,96 & 0,65 & 0,76 & 5,22 & 7,60 & 12,68 \\
\hline Tabasco & 0,36 & 0,96 & 1,07 & 5,43 & 7,82 & 4,57 \\
\hline Tamaulipas & 0,66 & 0,85 & 0,62 & 4,75 & 6,89 & 9,57 \\
\hline Tlaxcala & 0,22 & 1,03 & 0,61 & 4,18 & 6,05 & 3,66 \\
\hline Veracruz & 0,31 & 0,88 & 0,41 & 5,92 & 7,53 & 4,15 \\
\hline Yucatán & 0,08 & 0,49 & 0,58 & 4,89 & 6,04 & 1,35 \\
\hline Zacatecas & 0,45 & 0,70 & 0,98 & 4,62 & 6,75 & 6,72 \\
\hline
\end{tabular}

Fuente: elaboración de los autores con base en datos del Sistema Nacional de Información en Salud (http://www.sinais. salud.gob.mx/).

trienios (cuadro 2). En 19 de las 32 entidades federativas del país, los AEVP por homicidios en 2008-2010 aumentaron con respecto a 1998-2000. Entre ambos períodos, 18 estados registraron reducciones en su esperanza de vida temporaria, y 14 de ellos registraron aumentos en los AEVP por homicidios. Asimismo, en 2008-2010 hubo cuatro estados con dos o más AEVP por homicidio, comparado con ninguno en 1998-2000.

Chihuahua, Durango, Sinaloa y Guerrero fueron, en ese orden, los estados con mayor disminución de la EVM entre 15 y 75 años de edad en el lapso estudiado, y también los que presentaron mayor número de AEVP por homicidio; Chihuahua es el caso más llamativo, pues la cifra de AEVP por homicidios rebasó los cinco años en 2008-2010 y el cambio absoluto entre ambos trienios fue de 4,4 años. Además, Chihuahua y Durango mostraron la EVM entre 15 y 75 años de edad más baja del país (48 y 50,9 años, respectivamente) en 2008-2010.

En estados como Aguascalientes y Nuevo León, que en 1998-2000 presentaron cifras reducidas de AEVP por homicidios, se observó un (rápido) aumento de los AEVP superior al 9\% anual. Por el contrario, también es evidente cómo en varios estados se redujo el número de AEVP por homicidios entre los hombres, destacando casos como Chiapas, Campeche y Querétaro, donde el ritmo de reducción de AEVP por homicidios fue superior al 4,5\% anual. En Querétaro y Chiapas se aprecia también un incremento de la EVM temporaria, que en ambos casos supera el valor observado a escala nacional. Por último, debe señalarse a Yucatán como el estado que en ambos trienios presentó el menor número de AEVP por homicidios entre $15 \mathrm{y}$ 75 años de edad.

Entre los 10 estados que presentan AEVP por homicidios masculinos por encima del promedio nacional en 20082010 se pueden definir, por su ubicación geográfica, dos grupos: seis estados localizados en el norte-noroeste del país (Baja California, Chihuahua, Durango, Nayarit, Sinaloa y Sonora), los dos primeros y el último fronterizos con Estados Unidos y cuatro estados en el centro-sur del país (Guerrero, Michoacán, Morelos y Oaxaca). Todos ellos comparten orografía - están atravesados por la Sierra de Baja California, la Sierra Madre Occidental o por la Sierra Madre del Sur-y se ubican en la vertiente del océano Pacífico (figura 1).

Por otra parte, se observa que en 20082010 los homicidios fueron la causa que generó el mayor porcentaje de AEVP masculinos entre 20 y 44 años de edad a nivel nacional, a diferencia de los accidentes de transporte -que tuvieron mayor peso porcentual antes de los 20 años de edad- y la diabetes, cuyo impacto proporcional fue mayor a partir de los 45 años de edad (figura 2). Al mismo tiempo, se pone de manifiesto que las muertes violentas consideradas en este estudio (homicidios y accidentes de transporte) contribuyeron con casi $40 \%$ de los AEVP masculinos en el grupo etario de 15 a 29 años.

Por último, el análisis de comportamiento por grupos de edad de los AEVP por homicidios masculinos en cada trienio revela que el incremento en AEVP entre ambos momentos se debió, en la práctica, al aumento observado en los AEVP en el grupo de 15 a 44 años de edad: mientras en 1998-2000 los AEVP en esas edades sumaban 0,45 y representaban el $78 \%$ de los AEVP por homicidios, en 2008-2010 sumaron 0,71 (un 58\% más) y representaron el $84 \%$ del total de AEVP por homicidios entre los 15 y 75 años (figura 3). Cabe destacar tanto el incremento proporcional de AEVP por homicidio entre 25 y 39 años de edad superior al $60 \%$ - como la disminución de los AEVP a partir de los 55 años.

\section{DISCUSIÓN}

Diversos autores han señalado a la violencia como un eje básico para entender la relación entre masculinidad y salud. Sin dudas, el modelo de mas- 
CUADRO 2. Años de esperanza de vida perdidos (AEVP) por causa de homicidio y esperanza de vida temporaria (EVT) en personas de entre 15 y 75 años, por estado, México, 1998-2000 y 2008-2010

\begin{tabular}{|c|c|c|c|c|c|c|c|}
\hline \multirow[b]{3}{*}{ Estado } & \multicolumn{4}{|c|}{ AEVP } & \multirow{2}{*}{\multicolumn{3}{|c|}{ EVT }} \\
\hline & & & \multicolumn{2}{|c|}{ Cambio } & & & \\
\hline & 1998-2000 & 2008-2010 & $\begin{array}{c}\text { Absoluto } \\
1998-00 / 2008-10\end{array}$ & $\begin{array}{c}\text { Relativo anual } \\
1998-00 / 2008-10(\%)\end{array}$ & 1998-2000 & 2008-2010 & $\begin{array}{c}\text { Diferencia } \\
1998-00 / 2008-10\end{array}$ \\
\hline México (todos los estados) & 0,58 & 0,84 & 0,26 & 3,71 & 52,77 & 52,82 & 0,05 \\
\hline Aguascalientes & 0,11 & 0,29 & 0,18 & 9,35 & 53,70 & 53,75 & 0,04 \\
\hline Baja California & 0,96 & 1,68 & 0,72 & 5,48 & 50,32 & 51,70 &,- 39 \\
\hline Baja California Sur & 0,26 & 0,27 & 0,01 & 0,51 & 53,09 & 53,25 & 0,16 \\
\hline Campeche & 0,49 & 0,26 & $-0,22$ & $-6,35$ & 53,56 & 53,55 & $-0,01$ \\
\hline Coahuila & 0,26 & 0,55 & 0,30 & 7,43 & 53,32 & 53,03 & $-0,29$ \\
\hline Colima & 0,42 & 0,58 & 0,15 & 3,03 & 53,09 & 53,30 & 0,21 \\
\hline Chiapas & 0,75 & 0,32 & $-0,43$ & $-8,98$ & 52,38 & 52,76 & 0,38 \\
\hline Chihuahua & 0,87 & 5,23 & 4,36 & 16,43 & 51,49 & 48,00 & $-3,49$ \\
\hline Distrito Federal & 0,44 & 0,44 & 0,00 & $-0,05$ & 52,81 & 53,55 & 0,74 \\
\hline Durango & 0,76 & 2,60 & 1,84 & 11,62 & 53,30 & 50,90 & $-2,41$ \\
\hline Estado de México & 0,76 & 0,55 & $-0,21$ & $-3,23$ & 52,73 & 53,63 & 0,89 \\
\hline Guanajuato & 0,27 & 0,38 & 0,11 & 3,34 & 53,28 & 53,20 & $-0,08$ \\
\hline Guerrero & 1,67 & 2,29 & 0,62 & 3,10 & 53,02 & 51,75 & $-1,26$ \\
\hline Hidalgo & 0,26 & 0,23 & $-0,03$ & $-1,24$ & 52,95 & 53,06 & 0,11 \\
\hline Jalisco & 0,39 & 0,48 & 0,09 & 2,15 & 53,01 & 53,19 & 0,18 \\
\hline Michoacán & 0,84 & 0,97 & 0,13 & 1,46 & 52,78 & 52,23 & $-0,55$ \\
\hline Morelos & 0,90 & 0,87 & $-0,03$ & $-0,36$ & 53,13 & 53,33 & 0,20 \\
\hline Nayarit & 0,65 & 1,38 & 0,73 & 7,21 & 53,68 & 52,57 & $-1,11$ \\
\hline Nuevo León & 0,12 & 0,47 & 0,35 & 12,71 & 53,88 & 53,52 & $-0,36$ \\
\hline Oaxaca & 1,22 & 0,87 & $-0,35$ & $-3,48$ & 51,74 & 52,37 & 0,64 \\
\hline Puebla & 0,43 & 0,29 & $-0,14$ & $-3,91$ & 51,93 & 53,09 & 1,15 \\
\hline Querétaro & 0,31 & 0,20 & $-0,11$ & $-4,60$ & 52,49 & 53,49 & 1,00 \\
\hline Quintana Roo & 0,38 & 0,43 & 0,05 & 1,20 & 53,99 & 53,49 & $-0,50$ \\
\hline San Luis Potosí & 0,55 & 0,48 & $-0,06$ & $-1,25$ & 53,80 & 53,72 & $-0,08$ \\
\hline Sinaloa & 1,02 & 2,80 & 1,77 & 9,58 & 53,30 & 51,70 & $-1,60$ \\
\hline Sonora & 0,52 & 0,96 & 0,45 & 6,07 & 52,39 & 52,39 & $-0,01$ \\
\hline Tabasco & 0,32 & 0,36 & 0,04 & 1,25 & 52,86 & 52,18 & $-0,68$ \\
\hline Tamaulipas & 0,41 & 0,66 & 0,24 & 4,53 & 53,29 & 53,12 & $-0,17$ \\
\hline Tlaxcala & 0,29 & 0,22 & $-0,07$ & $-2,88$ & 53,57 & 53,95 & 0,38 \\
\hline Veracruz & 0,32 & 0,31 & 0,00 & $-0,09$ & 52,66 & 52,46 & $-0,20$ \\
\hline Yucatán & 0,08 & 0,08 & 0,00 & $-0,40$ & 54,06 & 53,96 & $-0,10$ \\
\hline Zacatecas & 0,40 & 0,45 & 0,06 & 1,34 & 53,80 & 53,24 & $-0,56$ \\
\hline
\end{tabular}

Fuente: elaboración de los autores con base en datos del Sistema Nacional de Información en Salud (http://www.sinais.salud.gob.mx/).

culinidad predominante en México y América Latina adjudica al varón el papel dominante, asignándole ciertos atributos —fortaleza, agresividad, competitividad, comportamientos violentos o temerarios, entre otros- social y culturalmente aceptados, pero que a largo plazo pueden representar un elevado costo para su salud $(29,30)$. En la práctica, ser hombre significa tener y ejercer autoridad y poder; en este medio, los jóvenes asumen desde temprana edad que la violencia no solo es un modo aceptable de resolver conflictos, sino que es una forma admirada y premiada de hacerlo $(31,32)$.

Desde esta perspectiva, es entendible la mayor implicación de los hombres, sobre todo jóvenes, en actividades delictivas de alto riesgo como poseer armas, participar en crímenes callejeros, participar en guerras de pandillas y cometer delitos relacionados con drogas, lo cual incrementa su probabilidad de morir en eventos de violencia: la tasa de hombres jóvenes víctimas de homicidio en el mundo es tres veces mayor que la tasa promedio mundial (33).

Es en este contexto donde debe evaluarse el impacto de los homicidios en el comportamiento de la EVM en México. En primer lugar, vale señalar que en congruencia con el relativo estancamiento de la EVM al nacer en México, durante el período de 10 años contemplado en el presente estudio la esperanza de vida en el grupo etario de 15 a 75 años apenas se incrementó en $0,1 \%$, alcanzando en 2008-2010 una cifra apenas mayor (52,34 años) a la calculada para este país en el bienio 1992-1993 (14).

Sin dudas, la EVM entre los 15 y los 75 años de edad podría haber crecido más en la década estudiada, si la tasa de homicidios masculina no hubiese aumentado en fechas recientes, elevando el número de AEVP por esta causa: el ritmo de crecimiento anual entre 1998-2000 y 2008-2010 de los AEVP por homicidios $(3,7 \%)$ es incluso mayor que por diabetes $(2,5 \%)$ y muy superior al de los accidentes de transporte $(0,3 \%)$.

Los AEVP por homicidio en 2008-2010 fueron 0,84. En Estados Unidos, Lemaire (13) — con un rango de edades y un método diferente al aquí empleadoestimó los días de esperanza de vida masculina perdidos por homicidios con armas de fuego en el año 2000 en 73 (o sea, 0,2 años); Salama (12), por su parte, refiere que la EVN masculina en 2002 se redujo en más de dos años en ciudades brasileñas como Recife, Río de Janeiro y São Paulo. En Cuba, los AEVP por homicidio en 2002 fueron 0,24 (15), y Boleda y Arriaga (14) estimaron en algo menos 
FIGURA 1. Años de esperanza de vida perdidos (AEVP) por homicidio en hombres de entre 15 y 75 años, por estado, México, 2008-2010

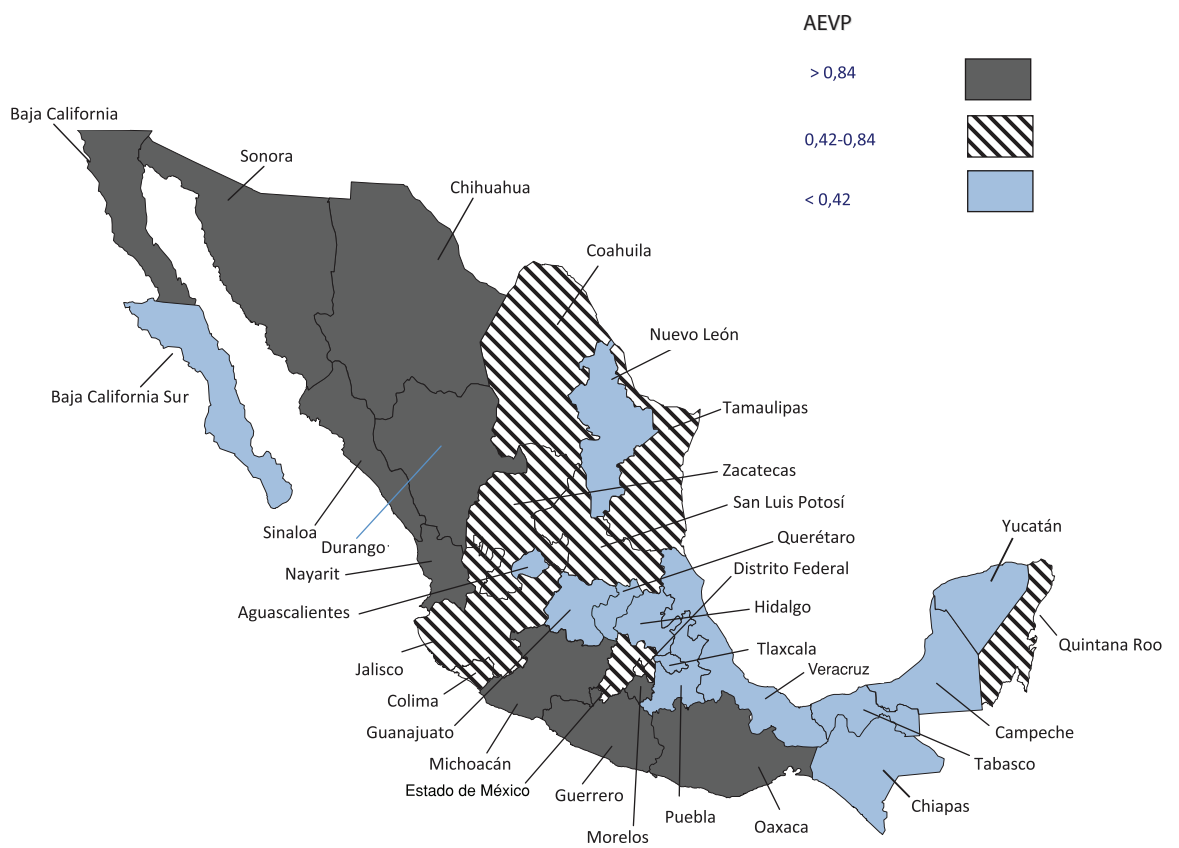

Fuente: elaboración de los autores con base en datos del Sistema Nacional de Información en Salud (http://www.sinais. salud.gob.mx/).

FIGURA 2. Años de esperanza de vida perdidos por homicidios, diabetes mellitus y accidentes de transporte, según grupos de edad, México, 2008-2010

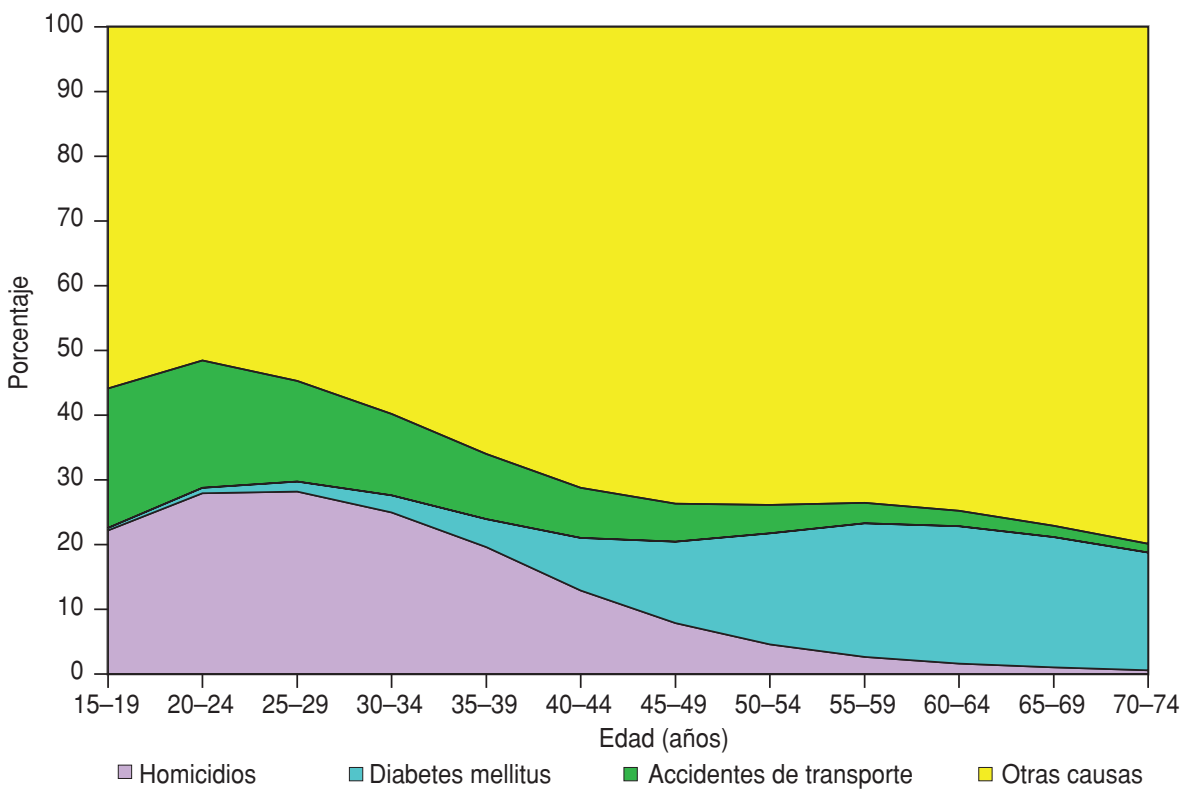

Fuente: elaboración de los autores con base en datos del Sistema Nacional de Información en Salud (http://www.sinais. salud.gob.mx/).

de un año los AEVP masculinos por homicidios y suicidios (considerados en conjunto) en México para 1992-1993, en cerca de medio año en Uruguay en 1990, y en alrededor de 0,4 años en Argentina y Chile para el mismo año. A fines de los años noventa, la Organización Panamericana de la Salud (OPS) estimó el número de AEVP por homicidios en México en alrededor de 0,5 (34), valor superior al de la mayoría de países latinoamericanos, pero cercano al calculado en el presente estudio para 1998-2000.

Con estos datos se puede afirmar que el número de AEVP por homicidio aquí estimado para 2008-2010 es similar al que presentaba México hace tres lustros, mayor al de hace una década y superior al de otros países latinoamericanos en años recientes, quizás con la excepción de ciertas ciudades de Brasil. A nivel estatal, la investigación revela importantes variaciones en cuanto a los AEVP por homicidio masculino. Dado que la utilización de este indicador posibilita establecer comparaciones entre poblaciones sin que estén influidas por la estructura de edades (26), las diferencias entre los estados deben ser explicadas por otros factores.

Estudios previos han establecido una asociación significativa entre actividades relacionadas con el narcotráfico — como el cultivo y la distribución de mariguana y opiáceos- y la tasa de homicidios a nivel estatal $(35,36)$. En general, la compleja orografía de varias entidades federativas ha favorecido estas actividades. Asimismo, en los estados del norte, dada su cercanía a Estados Unidos, se han asentado algunos de los grupos criminales más poderosos del país ligados al tráfico de drogas y a la introducción ilegal de armas de fuego, como los cárteles de Juárez, Tijuana, Sinaloa o los "Zetas", por citar algunos, los cuales han mantenido una dura lucha por el control de territorios $(37,38)$ que ha ocasionado un alto número de homicidios, en su mayoría masculinos.

Junto a esta realidad, factores tales como los altos niveles de impunidad y de corrupción existentes en los sistemas de seguridad y justicia (39-41), el elevado número de jóvenes de entre 15 y 29 años de edad que ni estudian ni trabajan (42) y en general el considerable porcentaje de población que padece algún tipo de pobreza o vulnerabilidad social (43) permiten comprender por qué, en estados como Chihuahua y Durango, el homicidio se ha convertido en el principal freno para el incremento de la EVM - o, en la práctica, ha coadyuvado a su reducción.

Cabe señalar que, aun cuando entre 2008 y 2010 las muertes masculinas por diabetes mellitus más que duplicaron a las provocadas por homicidios, su impacto en la EVM nacional fue similar, dado que las víctimas de homicidios son 
FIGURA 3. Años de esperanza de vida perdidos por homicidio, según grupos de edad, México, $1998-2000$ y 2008-2010

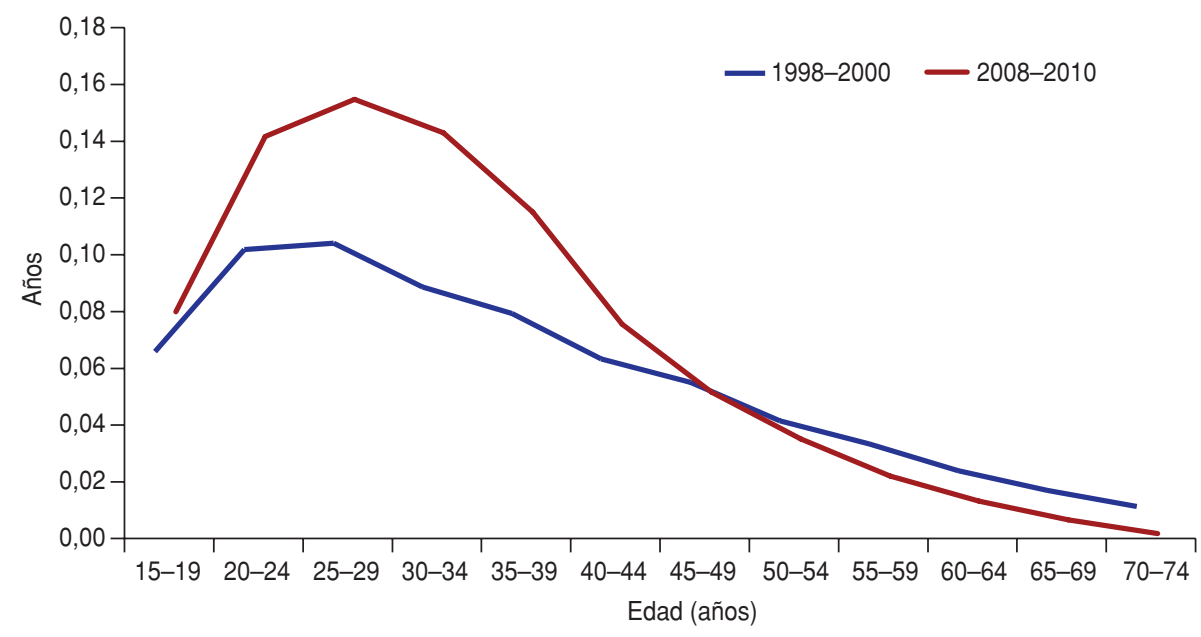

Fuente: elaboración de los autores con base en datos del Sistema Nacional de Información en Salud (http://www.sinais. salud.gob.mx/).

en buena medida jóvenes: alrededor de $27 \%$ de los AEVP en el grupo de entre 15 y 29 años de edad son imputables a los homicidios, mientras que los AEVP por diabetes comienzan a predominar a partir de los 45 años de edad.

Más todavía, si a los homicidios se les agregan las defunciones provocadas por accidentes de transporte, esta combinación causal sería responsable de 1,4 AEVP entre 2008 y 2010 —casi 0,6 más que los AEVP por diabetes mellitus- $y$ de aproximadamente 5 de cada 10 AEVP entre los varones de 15 a 29 años de edad en México, lo que ayuda a entender mejor la trascendencia de las muertes violentas estudiadas en el presente trabajo.

A su vez, el incremento en los AEVP por homicidios a nivel nacional entre ambos trienios se debió en gran parte al aumento de los AEVP por esta causa entre los 15 y 44 años de edad, reflejo del crecimiento de las tasas de homicidio masculino en las edades más jóvenes en años recientes (35). Estos datos demuestran que el homicidio masculino en México, al igual que en muchos otros países latinoamericanos, es un fenómeno principalmente juvenil (44), resultado de un conjunto de factores - entre ellos el narcotráfico, la impunidad y la falta de empleo o de plazas para continuar los estudios- que crean un clima de violencia e inseguridad que afecta en mayor grado a este grupo de población, aunque no con la misma intensidad en las diferentes regiones (42).
Este estudio presenta ciertas limitaciones. En primer lugar, el subregistro de defunciones existente en diferentes áreas de México - particularmente en las más marginadas $(45,46)$ - podría haber influido en la construcción de las tablas de mortalidad, y por ende en el cálculo de los AEVP; sin embargo, no existen datos probatorios de que en el caso de los homicidios $\mathrm{u}$ otras causas aquí analizadas haya un subregistro -en alguno de los estados o trienios analizados- lo suficientemente importante como para afectar de forma significativa los resultados; por el contrario, hay signos de avance en la calidad de los registros de la mortalidad de México $(34,47)$. Aun así, no puede soslayarse la posibilidad de una contabilización incompleta de los homicidios que resultan de enfrentamientos armados entre grupos del crimen organizado, o entre estos grupos y las fuerzas de seguridad, en cuyo caso los AEVP por homicidio, sobre todo en 2008-2010, podrían estar cuantitativamente subestimados.

Otra posible limitación en el uso de los AEVP es que se requiere acotar las edades que se analizan. En este caso, al dejar fuera del análisis a los varones menores de 15 años y a los mayores de 75 , el número de AEVP es menor que si se analizara un intervalo más amplio, en el cual se incluyera a casi todas las muertes por las causas estudiadas. Contrariamente, al escoger el intervalo de 15-75 años se puede evaluar mejor el impacto de los homicidios en la esperanza de vida en aquellas edades donde este fenómeno tiene una mayor frecuencia.

Por último, si bien hay tres posibles supuestos a considerar para el cálculo de AEVP, cualquiera que se utilice no modifica la tendencia de los resultados ni altera de modo sustancial las cifras de AEVP. El supuesto de mortalidad nula es recomendado en la literatura $(27,48)$, pues no solo facilita la interpretación sino que también explica en su totalidad los cambios de las esperanzas de vida temporarias por edad y causas de muerte.

\section{Conclusiones}

Los resultados del presente estudio sugieren que la EVM en México podría incrementarse si se lograran reducir los altos niveles de violencia homicida que existen en el país, máxime aquellos que involucran a hombres jóvenes. Dado que la EVN ha alcanzado cifras relativamente elevadas y que su crecimiento se ha ralentizado en años recientes, la reducción de las tasas de homicidios - sobre todo en la población joven- debería traducirse en una mayor esperanza de vida para los hombres mexicanos. Ahora bien, en ciertos estados del país donde la EVM ha descendido, como Chihuahua y Durango, el lograr que disminuya la violencia homicida se torna perentorio si se quiere retornar a los valores de esperanza de vida alcanzados ya a finales del decenio de los noventa. Dicho esto, algunos resultados positivos observados en varias entidades del país demuestran que la reducción de AEVP por homicidios es posible y tiene un impacto positivo en la EVM.

\section{Recomendaciones}

Analizar la violencia desde una perspectiva epidemiológica (49) puede ayudar a disminuir los homicidios $\mathrm{y}$, por ende, posibilitar que los hombres ganen más años de esperanza de vida. Estos objetivos implican implementar políticas públicas cuyo diseño tome en cuenta las características de la violencia en las diferentes regiones del país, así como los factores de riesgo que la pueden potenciar. Requieren además combinar acciones preventivas y punitivas concordantes con las realidades de los diferentes territorios del país, toda vez que los esfuerzos de carácter local o regional 
pueden arrojar mejores resultados en la lucha contra la violencia homicida que el establecimiento de políticas nacionales que no se ajusten a cada situación concreta $(49,50)$. Un ejemplo podría más restrictivas para evitar el ingreso ilegal de armas de fuego al país, sobre todo en la frontera norte, las cuales han demostrado ser efectivas en otros países (21). ser la adopción de políticas y medidas

Estas políticas deben basarse en evidencias lo más sólidas posible. En tal sentido, el uso en el presente estudio de un indicador como los AEVP - poco utilizado en el ámbito nacional- agrega un valor estratégico al análisis de los cambios de la mortalidad por homicidios a lo largo del tiempo, y ofrece a quienes toman decisiones pruebas concretas sobre el impacto del homicidio en la salud de los hombres mexicanos.

\section{REFERENCIAS}

1. Jackson R, Strauss R, Howe N. El desafío del envejecimiento en América Latina. Washington, DC: Center for Strategic and International Studies; 2009.

2. Mina A. Evolución de la mortalidad: pasado, presente y futuro. En: García B y Ondorica M, eds. Los grandes problemas de México: Población. México, D.F.: El Colegio de México; 2010. Pp. 79-104.

3. México, Instituto Nacional de Estadística y Geografía (INEGI). Mujeres y hombres en México 2010. Aguascalientes, México: INEGI; 2010.

4. Escalante-Gonzalbo F. Homicidios 2008-2009: La muerte tiene permiso. Nexos. 2011;397. Disponible en: http://www.nexos.com. $\mathrm{mx} /$ ?P=leerarticulo\&Article $=1943189$ Acceso el 31 de octubre de 2012.

5. Organización de Estados Americanos (OEA). Observatorio de Seguridad Ciudadana - Repositorio de Datos. México: Criminalidad y Violencia. Disponible en: http://www.oas. org/dsp/observatorio/database/coun triesdetails.aspx?lang=es\&country=MEX Acceso el 31 de octubre de 2012.

6. Organización de Estados Americanos (OEA). Observatorio de Seguridad Ciudadana - Repositorio de Datos. Colombia: Criminalidad y Violencia. Disponible en: http://www.oas. org/dsp/observatorio/database/coun triesdetails.aspx?lang=es\&country $=\mathrm{COL}$. Acceso el 31 de octubre de 2012.

7. Estados Unidos, Departamento de Justicia - Oficina Federal de Investigación de Es tados Unidos (FBI). Crime in the United States, 2009. Disponible en: http://www2. fbi.gov/ucr/cius2009/offenses/expan ded_information/data/shrtable_07.html Acceso el 31 de octubre de 2012

8. Canada, Statistics Canada. Homicides in Canada, 2009. Disponible en: http://www. statcan.gc.ca/pub/85-002-x/2010003/ article/11352-eng.htm\#a6 Acceso el 31 de octubre de 2012.

9. Rodríguez F. La pobreza como un fenómeno de violencia estructural. Rev Cienc Soc. 2004;10(1):42-50.

10. Souza ER, Minayo MC, Silva CM, Reis AC, Malaquias JV, Veiga JP, et al. Analise Temporal da Mortalidade por Causas Externas no Brasil: Décadas de 80 e 90. En: Minayo MC, Souza ER, eds. Violência sob o Olhar da Saúde. Rio de Janeiro: Fiocruz; 2006. Pp.: 83-107.

11. Cardona M, García HI, Giraldo CA, López MV, Suárez CM, Corcho DC, et al. Homicidios en Medellín, Colombia, entre 1990 y 2002: actores, móviles y circunstancias. Cad Saude Publica. 2005;21(3):840-51.

12. Salama P. Informe sobre la violencia en América Latina. Revista de Economía Institucional. 2008;10(18):81-102

13. Lemaire J. The cost of firearms death in the United States: Reduced life expectancies and increase insurance costs. J Risk Insur. 2005;72(3):359-74.

14. Boleda M, Arriaga E. América Latina: Mortalidad por accidentes y por violencia contra las personas. Notas Poblacion. 2000;28(70): 87-119.

15. Cuba, Oficina Nacional de Estadística, Centro de Estudios en Población y Desarrollo. La esperanza de vida en Cuba, 2001-2003. La Habana: Fondo de Población de Naciones Unidas; 2004

16. México, Dirección General de Información en Salud (DGIS). Base de datos de defunciones 1979-2010. Sistema Nacional de Información en Salud (SINAIS). Disponible en: http://www.sinais.salud.gob.mx Acceso el 17 febrero 2012.

17. México, Dirección General de Información en Salud (DGIS). Base de datos de estimaciones de población, 1990-2012, El Colegio de México. Sistema Nacional de Información en Salud (SINAIS). Disponible en: http://www.sinais.salud.gob.mx Acceso el 15 octubre 2011.

18. Organización Panamericana de la Salud (OPS). Clasificación Estadística Internacional de Enfermedades y Problemas relacionados con la Salud. Publicación Científica No. 554, Vol. 1. Washington, DC: OPS; 1995.

19. Rodríguez García J. Desigualdades socioeconómicas entre departamentos y su asociación con indicadores de mortalidad en Colombia en 2000. Rev Panam Salud Publica. 2007;21(2/3):111-24.

20. Lozada P, Aguinaga L, Páez R, Olmedo C, Pozo A. El peso de la enfermedad en el Ecuador. Quito: Centro de Estudios de Población y Desarrollo Social (CEPAR); 1999.

21. Moreno C, Cendales R. Mortalidad y años potenciales de vida perdidos por homicidios
Finalmente, hacen falta medidas y acciones urgentes que contribuyan efectivamente a revertir ciertos valores que por tradición se asocian al concepto hegemónico de masculinidad, transmitidos desde el comienzo mismo del proceso de socialización del individuo, que llevan implícito un estilo de vida que involucra a los hombres con mayor frecuencia que a las mujeres en actos violentos y los expone a un mayor riesgo de muerte prematura. en Colombia, 1985-2006. Rev Panam Salud Publica. 2011;30(4):342-53.

22. Särndal C, Swensson B, Wretman J. Basic Ideas in Estimation from Probability Samples. In: Särndal C, Swensson B, Wretman J, eds. Model Assisted Survey Sampling. New York: Springer-Verlag; 1997. Pp. 24-58.

23. Coale A, Demeny P. Regional Model Life Tables and Stable Populations. Princeton, New Jersey: Princeton University Press; 1966

24. Organización Panamericana de la Salud (OPS). Bases Conceptuales Demográficas. Nicaragua, 2010. Disponible en: http://new. paho.org/nic/index.php?option $=$ om doc man\&task $=$ doc_details\&gid $=45 \&$ Item $\mathrm{id}=235$ Acceso el 7 de noviembre de 2012.

25. Organización Panamericana de la Salud (OPS). Sobre la estimación de tasas de mortalidad para países de la región de las Américas. Boletín Epidemiológico. 2003;24(4):1-5. Disponible en: http://www.paho.org/spanish/ dd/ais/be_v24n4-mortalidad.htm Acceso el 31 de octubre de 2012.

26. Arriaga E. Los años de vida perdidos: su utilización para medir el nivel y el cambio de la mortalidad. Notas Poblacion; 1996;24(63):7-38.

27. Arriaga E. Comentarios sobre algunos índices para medir el nivel y el cambio de la mortalidad. Estud Demogr Urbanos Col Mex. 1996;11:5-30.

28. Xunta de Galicia, Organización Panamericana de la Salud. EPIDAT 3.1. Análisis epidemiológico de datos tabulados. A Coruña, Washington, DC; Xunta de Galicia, OPS; 2006.

29. De Keijzer B. Hasta donde el cuerpo aguante: género, cuerpo y salud masculina. En: Cáceres $C$, Cueto M, Ramos M, Vallenas S, eds. La salud como un derecho ciudadano. Perspectivas y propuestas desde América Latina. Lima, Perú: International Forum for Social Sciences in Health/Universidad Peruana Cayetano Heredia; 2003. Pp. 137-52.

30. Kimmel M. La producción teórica sobre la masculinidad: nuevos aportes. En: Rodríguez $\mathrm{R}$, ed. Fin de siglo: género y cambio civilizatorio. Ediciones de las Mujeres, No. 17. Santiago, Chile: Isis Internacional; 1992 . Pp. 129-38.

31. Kimmel M. Snips and Snails ... and Violent Urges. Newsday. 2001;8:A41-4.

32. Sotomayor Z, Román R. Masculinidad y violencia homicida. México DF: Plaza y Valdés; 2007. 
33. United Nations Office on Drugs and Crime (UNODOC). 2011 Global Study on Homicide. Trends, Contexts, Data. Disponible en: http:/ / www.unodc.org/southerncone/ es/frontpage/2011/10/06-global-studyon-homicide-2011.html Acceso el 1 de noviembre de 2012.

34. Organización Panamericana de la Salud (OPS). La Salud en las Américas. Ed 2002, Volumen I. Publicación Científica y Técnica no. 587. Washington DC: OPS; 2002.

35. González-Pérez GJ, Vega-López MG, VegaLópez A, Muñoz-de-la-Torre A, CabreraPivaral CE. Homicidios de adolescentes en México, 1979-2005: evolución y variaciones sociogeográficas. Papeles Poblac. 2009;15(62):109-41.

36. González-Pérez GJ, Vega-López MG, Cabrera-Pivaral CE, Vega-Lopez A. Violence and health: an epidemiological analysis of homicides in México, 1979-2008. J Epidemiol Community Health. 2011;65:A187.

37. González L. Los cárteles más fuertes de México. Disponible en: http://www.fp-es. org / lista-los-carteles-mas-fuertes-demexico Acceso el 1 de noviembre de 2012.

38. Astorga L. Seguridad, traficantes y militares. (El poder y la sombra.) Tiempo de Memoria. México DF: Tusquets; 2007.

39. González L. El crecimiento del narco no es posible sin la complicidad de las autoridades. Disponible en: http://www.fp-es.org/el- crecimiento-del-narco-no-es-posible-sinla-complicidad-de-las-autoridades Acceso el 1 de noviembre de 2012.

40. Aguilar-Camín H. Detenidos y deudores. Milenio. Disponible en: http://www.milenio.com/cdb/doc/impreso/9050204 Acceso el 1 de noviembre de 2012.

41. Damián F. García Luna: aporta el crimen 14 mil mdp a salario policial. Milenio. Disponible en: http://www.milenio.com/cdb/ doc/impreso/9043163 Acceso el 1 de noviembre de 2012.

42. Organización para la Cooperación y el Desarrollo Económico (OCDE). Panorama de la educación 2011. Nota de país-México. Disponible en: http://www.oecd.org/ dataoecd/32/32/48667648.pdf Acceso el 01 de noviembre de 2012.

43. México, Consejo Nacional de Evaluación de la Política de Desarrollo Social (CONEVAL). Metodología de medición multidimensional de la pobreza. Disponible en: http://www. coneval.gob.mx/contenido/home/6595 Acceso el 1 de noviembre de 2012.

44. Gomáriz Moraga E. La devastación silenciosa: jóvenes y violencia social en América Latina. San José, Costa Rica: FLACSO; 2011.

45. Freyermuth-Enciso G, Cárdenas-Elizalde R. Evaluación del subregistro de la mortalidad materna en Los Altos de Chiapas mediante las estrategias RAMOS y RAMOS modificada. Salud Publica Mex. 2009;51(6):450-7.
46. Lozano-Ascencio R. ¿Es posible seguir mejorando los registros de las defunciones en México? Gac Med Mex. 2008;144(6):525-33.

47. Mathers C, Ma Fat D, Inoue M, Rao Ch, Lopez A. Counting the death and what they died from: an assessment of the global status of cause of death data. Bull World Health Organ. 2005:83(3):171-7.

48. Bocco M. La relación entre los años de vida perdidos y la esperanza de vida: aplicaciones para el análisis de la mortalidad. Notas Poblacion. 1996;24:39-60.

49. Morrison A, Buvinic M, Shifter M. América violenta: Factores de riesgo, consecuencias e implicaciones para las políticas sobre violencia social y doméstica. En: Fruhling $\mathrm{H}$ Tulchin JS, Golding H, eds. Crimen y violencia en América Latina. Bogotá: Fondo de Cultura Económica; 2005. Pp. 117-54.

50. Volokh A, Snell L. School violence prevention: Strategies to keep schools safe. Reason Public Policy Institute. Policy Study No. 234. Los Angeles: Reason Public Policy Institute; 1998.

Manuscrito recibido el 16 de noviembre de 2011. Aceptado para publicación, tras revisión, el 9 de septiembre de 2012.

ABSTRACT Objective. To determine the impact of homicide on male life expectancy in Mexico and its 32 states during the three-year periods 1998-2000 and 2008-2010 and the weight of the different age groups in years of life expectancy lost (YLEL) due to this cause.

\section{Impact of homicide on male life expectancy in Mexico}

Methods. Based on official death and population data, abridged tables for male mortality in Mexico as a whole and its states were created for the three-year periods studied. Health-adjusted life expectancy and YLEL for men aged 15 to 75 were calculated by selected causes (homicide, diabetes mellitus, and traffic accidents) and age groups in each three-year period.

Results. In the years between the 1998-2000 and 2008-2010 periods, YLEL due to homicide increased both nationally and in 19 states. In four states, the YLEL in 2008-2010 exceeded two, with the state of Chihuahua standing out at 5.2 years. In 14 of the 18 states where health-adjusted life expectancy among men declined between the two three-year periods, the YLEL due to homicide increased. From 2008 to 2010, homicides were the leading cause of YLEL among men aged 20-44. YLEL due to homicide among those aged 15-44 increased between the two three-year periods. Conclusions. The increase in the rate of homicidal violence, especially among young people, is impeding an increase in male life expectancy in Mexico. In several states, such as Chihuahua and Durango, this violence appears to be the main reason for the decline in life expectancy among men aged 15 to 75 .

Key words Violence; homicide; mortality; life expectancy; Mexico. 